ARTICLE

Received 28 Nov 2015 | Accepted 15 Jun 2016 | Published 20 Jul 2016

\title{
High-speed ultrasound imaging in dense suspensions reveals impact-activated solidification due to dynamic shear jamming
}

\author{
Endao Han ${ }^{1,2}$, Ivo R. Peters ${ }^{1,3} \&$ Heinrich M. Jaeger ${ }^{1,2}$
}

A remarkable property of dense suspensions is that they can transform from liquid-like at rest to solid-like under sudden impact. Previous work showed that this impact-induced solidification involves rapidly moving jamming fronts; however, details of this process have remained unresolved. Here we use high-speed ultrasound imaging to probe non-invasively how the interior of a dense suspension responds to impact. Measuring the speed of sound we demonstrate that the solidification proceeds without a detectable increase in packing fraction, and imaging the evolving flow field we find that the shear intensity is maximized right at the jamming front. Taken together, this provides direct experimental evidence for jamming by shear, rather than densification, as driving the transformation to solid-like behaviour. On the basis of these findings we propose a new model to explain the anisotropy in the propagation speed of the fronts and delineate the onset conditions for dynamic shear jamming in suspensions.

\footnotetext{
${ }^{1}$ James Franck Institute, The University of Chicago, Chicago, Illinois 60637, USA. ${ }^{2}$ Department of Physics, The University of Chicago, Chicago, Illinois 60637 USA. ${ }^{3}$ Engineering and the Environment, University of Southampton, Highfield, Southampton SO17 1BJ, UK. Correspondence and requests for materials should be addressed to H.M.J. (email: h-jaeger@uchicago.edu).
} 
$\mathrm{D}$ ense suspensions are complex fluids that can exhibit strong, discontinuous shear thickening, where the viscosity jumps up orders of magnitude when a critical shear stress is exceeded ${ }^{1-4}$. Under a wide range of dynamic conditions, dense suspensions can also undergo a transformation to solid-like behaviour, for example, during sudden impact at their free surface $^{5-7}$, ahead of quickly sinking objects ${ }^{8,9}$, under shear ${ }^{10}$ or during rapid extension ${ }^{11}$. Detailed investigation of the dynamics during impact has shown how such solidification is associated with a propagating front that converts fluid-like, unjammed suspension into rigidly jammed material in its wake $e^{5,12}$. This dynamic jamming front moves through the suspension with a speed much greater than the impactor itself.

To explain this solidification, a model was proposed ${ }^{5}$ that assumed the impact pushes the particles closer together until they jam. This densification scenario was based on the standard jamming phase diagram for frictionless hard particles, where entry into a jammed state requires an increase in particle packing fraction $\phi$ (ref. 13). Since the volume of particles is conserved, the front propagation speed $v_{\mathrm{f}}$ along the direction of impact is then related to the impactor speed $v_{\mathrm{p}}$ via ${ }^{14}$

$$
v_{\mathrm{f}}=\frac{\phi_{\mathrm{J}}}{\phi_{\mathrm{J}}-\phi_{0}} v_{\mathrm{p}},
$$

where $\phi_{\mathrm{J}}$ is the packing fraction at which jamming occurs and $\phi_{0}<\phi_{\mathrm{J}}$ is the packing fraction of the initially unjammed suspension at rest. The closer the initial packing fraction is to jamming, the faster the front will propagate, in principle diverging at $\phi_{\mathrm{J}}$. This model shows excellent agreement with measurements of $v_{\mathrm{f}}$ in systems where the local packing fraction can change easily, such as dry granular particle layers that are being compacted snowplough-like from one end ${ }^{14}$.

In suspensions, the presence of an interstitial liquid makes it possible to prepare three-dimensional (3D) systems at packing fractions $\phi$ well below $\phi_{\mathrm{J}}$ by density matching the particles to the liquid. Given that such systems can still exhibit impact-induced solidification, jamming by densification would imply significant particle packing fraction changes $\Delta \phi=\phi_{\mathrm{J}}-\phi$. However, unless the impact speed is so high that the liquid becomes compressible ${ }^{15}$, viscous drag will counteract any densification of the particle sub-phase. This calls into question the mechanism underlying equation (1), even though there is experimental evidence for the basic outcome, namely that the ratio $v_{\mathrm{f}} / v_{\mathrm{p}}$ increases dramatically as $\Delta \phi$ approaches zero ${ }^{5,12}$.

One intriguing alternative mechanism has recently emerged with the concept of jamming by shear ${ }^{3,16}$. In this extension of frictionless, standard jamming, the presence of frictional interactions between particles makes it possible to start from initially isotropic, unjammed configurations at $\phi=\phi_{0}<\phi_{\mathrm{J}}$ and, without changing $\phi$, rearrange the particles into anisotropic fragile or jammed configurations by applying shear. Shear jamming is also possible in frictionless systems, albeit over a much smaller range in $\Delta \phi$ (ref. 17). So far, such shear jamming has been observed experimentally in two-dimensional (2D) dry granular systems under quasi-static conditions, where there is direct visual access to particle positions and stresses by imaging perpendicular to the $2 \mathrm{D}$ plane $\mathrm{e}^{16}$. Investigating the role of shear jamming in dynamic impact-induced solidification of 3D suspensions requires the capability of non-invasively tracking the jamming fronts and the associated, quickly evolving flow field in the interior of an optically opaque system.

Here we achieve this with ultrasound. Related methods have been applied to studying dry granular materials ${ }^{18,19}$ and steadystate flow in suspensions ${ }^{20,2}$. Measuring the speed of sound $c$ we obtain an upper bound on the change of packing fraction $\Delta \phi$ as the suspension jams. We find that at $v_{\mathrm{p}}<<c, \Delta \phi$ is much smaller than required if densification was the primary driver for impactactivated solidification. To investigate the crossover as $v_{\mathrm{p}}$ increases, we use high-speed ultrasound imaging to track the emergence of concentrated shear bands at the location of the propagating jamming fronts. In the regime of small $v_{\mathrm{p}}$, the suspension responds to stress like a fluid, and in the regime of large $v_{\mathrm{p}}$, the suspension develops solid-like characteristics, which we identify by investigating the flow fields. The invariant packing fraction and existence of shear bands provides direct evidence of dynamic shear jamming in 3D suspensions. Furthermore, access to the full flow field allows us to extract the local shear rates and identify the origin of a key, but so far unexplained, feature of the response to impact: the longitudinal front propagation speed exceeds the transverse propagation speed by a factor very close to two ${ }^{12}$.

\section{Results}

Experimental set-up and extraction of the flow field. The experiments were performed with a model suspension: cornstarch particles dispersed in water-glycerol $\mathrm{CsCl}$ solutions. The experimental set-up is illustrated in Fig. 1. In the impact experiments the impactor was driven vertically downward with constant velocity $v_{\mathrm{p}}$ by a linear actuator. A representative flow field $\left(u_{r}, u_{z}\right)$ inside the suspension during an impact is shown in Fig. 2a-c. The vertical and horizontal axes in the image correspond to the $z$ and $r$ directions in cylindrical coordinates. The flow field shows a jammed solid-like plug in the centre, as evidenced by the fact that all points move vertically with speed close to $v_{\mathrm{p}}$. Also evident is a strong velocity gradient around the periphery of the jammed region. To show this more explicitly, we calculate the local shear rate from the velocity field $\left(u_{r}, u_{z}\right)$. Given rotational symmetry, the shear rate tensor becomes

$$
\dot{\varepsilon}=\left[\begin{array}{ccc}
\frac{\partial u_{r}}{\partial r} & 0 & \frac{1}{2}\left(\frac{\partial u_{r}}{\partial z}+\frac{\partial u_{z}}{\partial r}\right) \\
0 & \frac{u_{r}}{r} & 0 \\
\frac{1}{2}\left(\frac{\partial u_{r}}{\partial z}+\frac{\partial u_{z}}{\partial r}\right) & 0 & \frac{\partial u_{z}}{\partial z}
\end{array}\right] .
$$

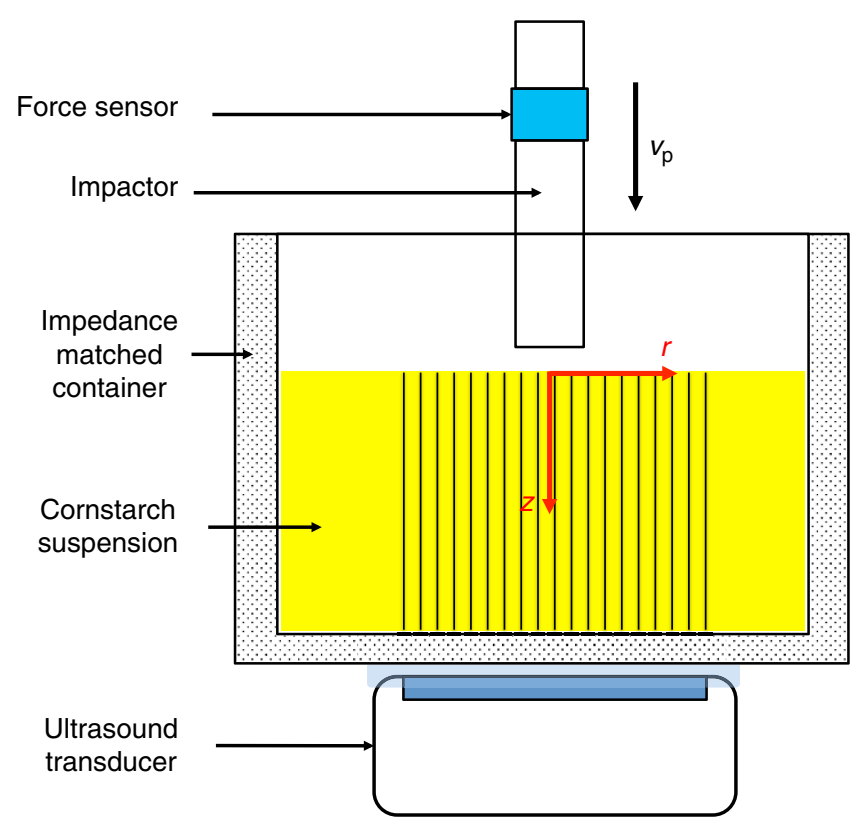

Figure 1 | Schematic illustration of the experimental set-up. The sample container and impactor are cylindrical and concentric. The ultrasound transducer scans a vertical slice centred along the central $z$ axis, providing a field of view as indicated by the striped area. 
a

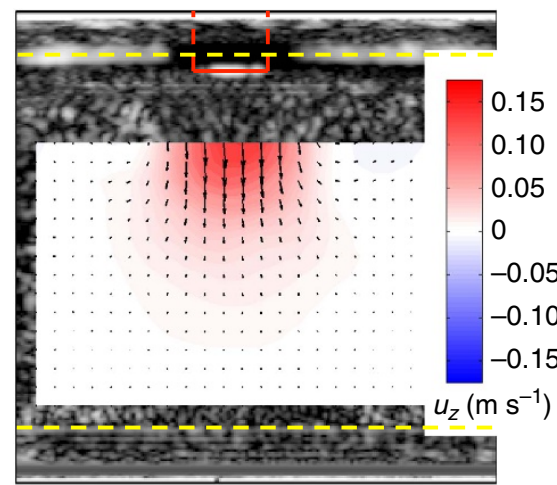

C

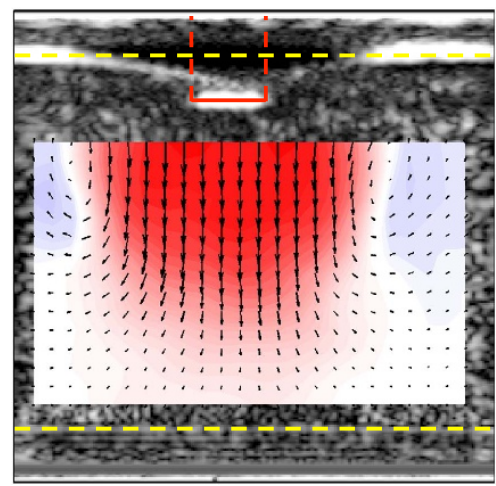

b

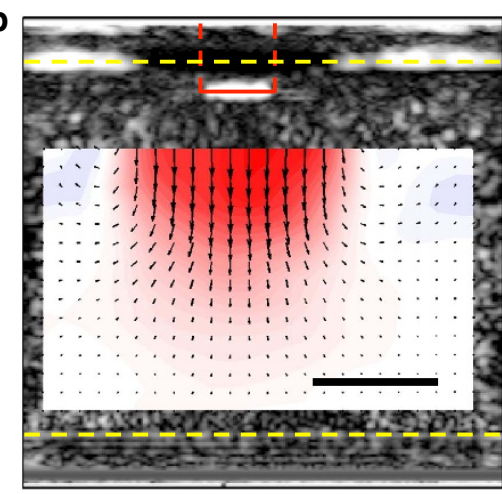

d

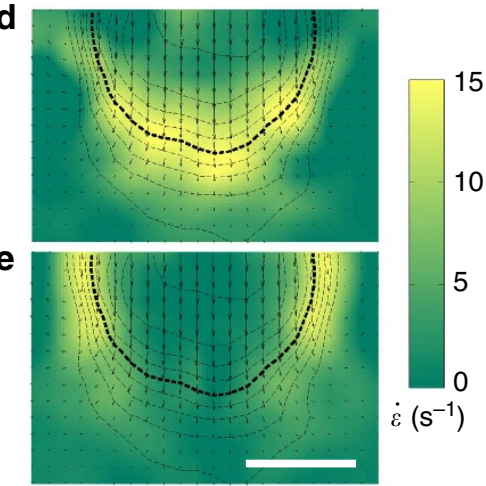

Figure 2 | Visualization of the flow field with ultrasound. (a-c) Velocity field during an impact at time $6.0 \mathrm{~ms}$ (a) $13.2 \mathrm{~ms}$ (b) and $20.3 \mathrm{~ms}$ (c) (the impactor reached the surface when time $t=0 \mathrm{~ms}$ ). The images are snapshots from a high-speed ultrasound movie (shown in grey scale) with overlaid velocity field from particle image velocimetry (PIV) analysis. The colour code represents the magnitude and sign of the vertical component of the local velocity $u_{z}$ (red corresponds to downward, blue to upward flow). Dashed yellow lines indicate the locations of the free surface of the suspension and of the bottom of the container. The impactor is outlined in red. The experimental parameters are $\phi=0.47, v_{\mathrm{p}}=175 \mathrm{~mm} \mathrm{~s}^{-1}$, liquid viscosity $\eta_{0}=4.6 \pm 0.2 \mathrm{mPa} \cdot \mathrm{s}$, fill depth $H=30 \mathrm{~mm}$, and impactor diameter of $6.0 \mathrm{~mm}$. The black scale bar in (b) represents $1 \mathrm{~cm}$ for $(\mathbf{a}-\mathbf{c})$. (d,e) Two components of the shear rate tensor, $\left|\dot{\varepsilon}_{z z}\right|$ (d) and $\left|\dot{\varepsilon}_{r z}\right|$ (e) shown for the same instant in time as the flow field in (c). Dashed lines are contours connecting points with the same $u_{z}$. The thicker line indicates $u_{z}=v_{\mathrm{p}} / 2$, which defines the front position. Scale bar, $(\mathbf{d}, \mathbf{e}) 1 \mathrm{~cm}$. The whole process is shown in Supplementary Movie 1.

Figure 2d,e shows the two components $\left|\dot{\varepsilon}_{z z}\right|=-\frac{\partial u_{z}}{\partial z}$ and $\left|\dot{\varepsilon}_{r z}\right|=-\frac{1}{2}\left(\frac{\partial u_{r}}{\partial z}+\frac{\partial u_{z}}{\partial r}\right)$. Underneath the jammed region, that is, in longitudinal direction, $\dot{\varepsilon}_{z z}$ dominates. This corresponds to pure shear that compresses the suspension in the $z$ direction and expands it radially. By contrast, along the sides of the jammed plug $\dot{\varepsilon}_{r z}$ dominates, and here the main contribution arises from the term $\frac{\partial u_{z}}{\partial r}$. As a result, the velocity gradient is mainly perpendicular to $v_{\mathrm{p}}$. This is analogous to simple shear as seen, for example, in parallel plate setups. We will return below to the implications of having both types of shear.

Speed of sound. In an unjammed suspension of solid particles in a Newtonian liquid, the shear modulus vanishes. In the limit that the solid particles are much smaller than the wavelength, the speed of sound is then given by $c=(K / \rho)^{\frac{1}{2}}$, where $K$ is the average bulk modulus and $\rho$ the average material density of the suspen$\operatorname{sion}^{22,23}$. In our experiments, the particles and suspending solvent are density matched (see Methods section), but the average $K$ still increases with $\phi$ since cornstarch particles have a bulk modulus larger than that of the liquid ${ }^{24}$. As shown in Fig. 3a, the resulting dependence of $c$ on $\phi$ is, to a good approximation, linear across the regime of packing fractions probed by our experiments. This allows us to obtain $\Delta \phi$ straightforwardly by detecting changes in $c$ with ultrasound.

A schematic illustration of the suspension under impact is shown in Fig. 3b, indicating two regions: a jammed region (orange) directly underneath the impactor and an unjammed region (yellow) ahead of the jamming front. Our measurements provide the average speed of sound $\bar{c}$ as determined from the time it takes sound pulses to traverse both regions (see Methods section). The process of transforming unjammed suspension to jammed suspension could be expected to increase the speed of sound via three possible mechanisms: (1) an increase $\Delta \phi$ in packing fraction; (2) an increase in effective bulk modulus $K$; (3) the development of a finite shear modulus $G$ as the suspension jams ${ }^{13}$. Currently, we cannot determine how much they each contribute, but we can estimate the upper limit of each individual term by assuming the other two zero.

First, we estimated the maximum possible increase in $\phi$. Figure $3 c$ shows the measured change in sound speed $\Delta c=\bar{c}-c\left(\phi_{0}\right)$ during impact for a suspension prepared at $\phi_{0}=0.48$. The impactor hits the suspension surface at time $t=0$, generating a jamming front that reaches the bottom at $t \approx 0.035 \mathrm{~s}$. We can identify this point by the dramatic increase in force on the impactor, as established by prior investigations of quasi-2D (ref. 12) and 3D (ref. 5) systems. Until the jamming front interacts with the bottom wall $\Delta c=\bar{c}-c\left(\phi_{0}\right)$ is less than the measurement uncertainty of about $5 \mathrm{~ms}^{-1}$. Neglecting any increases in $K$ and $G$, we find from Fig. 3a that our noise floor $\Delta c \approx 5 \mathrm{~m} \mathrm{~s}^{-1}$ implies $\Delta \phi \approx 0.006$ during the free propagation of the fronts. This means that $\phi$ could have increased to 0.49 at best. On the other hand, even at the highest packing fraction in Fig. 3a $\phi=0.52$, the suspension can still flow when sheared slowly, 

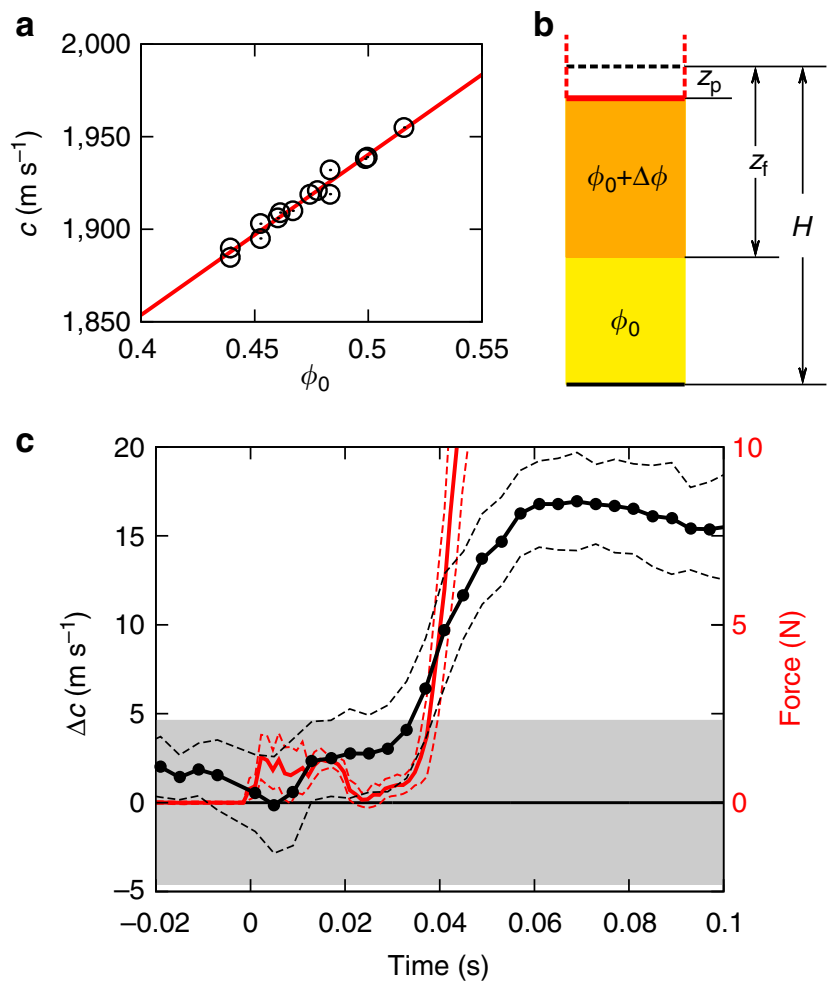

Figure 3 | Direct measurement of packing fraction changes. (a) Speed of sound $c$ as a function of packing fraction $\phi$. All the data were taken with suspensions in their quiescent fluid-like state, at packing fractions well below $\phi_{\text {J }}$. From the scattering of the data points we can see the overall uncertainty of this experiment. (b) Sketch of the region beneath the impactor. The black dashed line represents the initial suspension surface at a fill height $H$ above the bottom of the container (bold black solid line). As the impactor (outlined in red) pushes down a vertical distance $z_{\mathrm{p}}$ the front (orange region) propagates a distance $z_{\mathrm{f}}$ (c) Change in sound speed $\Delta c$ as a function of time (black trace) at $\phi_{0}=0.48$. Impact at the free suspension surface occurs at $t=0 \mathrm{~ms}$. Once the jamming front has reached the bottom of the container, the suspension below the impactor has been transformed into a solid-like material. At that point, near $t=35 \mathrm{~ms}$, the force on the impactor (red trace) rises markedly. Note that within our experimental uncertainties, the speed of sound does not increase until the shear-jammed region becomes compressed. Data are averages from seven experiments that simultaneously measured force and sound speed as functions of time. Dashed lines indicate 1 s.d. The grey region shows the uncertainty (given by 1 s.d.) in determining $\Delta c$ at low $v_{p}$, where no solidification takes place.

implying an isotropic jamming threshold $\phi_{\mathrm{J}}>0.52$. This means that the increase in packing fraction due to impact is much less than required for jamming by the densification model.

If instead we assume $\Delta \phi=0$ and use $c=\left[\left(K+\frac{4}{3} G\right) / \rho\right]^{\frac{1}{2}}$, as appropriate for solids ${ }^{25}$, to describe the dependence of the speed of sound on $K$ and $G$ within the jammed region behind the front, the same noise floor $\Delta c \approx 5 \mathrm{~m} \mathrm{~s}^{-1}$ implies that the net increase in the sum of the moduli $\tilde{K}=K+\frac{4}{3} G$ could not have been larger than $\Delta \tilde{K} \approx 32 \mathrm{MPa}$. This is very small compared with the bulk modulus $K_{0}$ of the quiescent suspension at $\phi_{0}=0.48$ : $\Delta \tilde{K} / K_{0} \approx 0.5 \%$.

Once the front has reached the bottom, $\Delta c$ increases to $\approx 16 \mathrm{~m} \mathrm{~s}^{-1}$. While this is significantly above the noise floor, it limits any packing fraction changes to $\Delta \phi \approx 0.02$, still less than necessary to reach $\phi_{\mathrm{J}}$. Nevertheless, the interaction with the solid boundary increases the stress in the dynamically jammed region significantly and this drives the suspension deeper into the shearjammed state. We can therefore expect concomitant increases in bulk and shear moduli and thus in sound speed. From the sound speed data in Fig. 3, the upper limit of the net increase in the sum of the moduli $\tilde{K}$ is $\Delta \tilde{K} \approx 101 \mathrm{MPa}$.

Propagation of fronts. From the evolution of the flow fields as shown in Fig. 2, we extract the position of the moving jamming front, defining the front position as the line of points where the vertical component of the impact velocity has dropped to $v_{\mathrm{p}} / 2$. In the following we focus on the points in the flow field that propagate the furthest in $z$ and $r$ directions, that is, on the maximum longitudinal and transverse front speeds. As shown in Fig. 4a, after an initial stage the fronts in both directions propagate essentially linearly as function of time before slowing down when they start to interact with boundaries and the incipient jammed region gets compressed by the impactor; further compression causes the motion to stop quickly (Supplementary Fig. 1). Here we investigate this linear regime, where the front propagates freely. To compare how quickly the front propagates relative to $v_{\mathrm{p}}$, we define two dimensionless front propagation factors, or normalized front speeds, $k$ as

$$
k_{\mathrm{t}}=\frac{v_{\mathrm{ft}}}{v_{\mathrm{p}}}, k_{\mathrm{l}}=\frac{v_{\mathrm{fl}}}{v_{\mathrm{p}}}-1
$$

where the subscripts $t$ and $l$ represent transverse and longitudinal directions, respectively. The ' -1 ' in $k_{1}$ compensates for the vertical motion of the impactor itself.

Our experiments show that the parameters that affect $k_{1}$ and $k_{\mathrm{t}}$ include $\phi, v_{\mathrm{p}}$ and the suspending liquid's viscosity $\eta_{0}$. For a suspension with given $\eta_{0}$ and $\phi_{0}$ that is impacted very slowly, the response is fluid like and both $k_{\mathrm{t}}$ and $k_{1}$ are close to zero. However, beyond a threshold value $v_{\mathrm{p} 0}$ jamming fronts start to appear. Their normalized speeds initially increase quickly with impactor speed $v_{\mathrm{p}}$ but eventually asymptote to a fixed $k^{*}$. The relation between $k_{1}$ and $v_{\mathrm{p}}$ in suspensions with the same $\eta_{0}$ but different $\phi_{0}$ is shown in Fig. $4 \mathrm{~b}$; the behaviour of $k_{\mathrm{t}}$ is similar. As $\phi$ increases, the curves shift towards lower $v_{\mathrm{p} 0}$ and higher $k^{*}$. For comparison, in suspensions with the same $\phi_{0}$, larger solvent viscosity $\eta_{0}$ leads to lower threshold $v_{\mathrm{p} 0}$ (Supplementary Fig. 2). To extract $k^{*}$ and $v_{\mathrm{p} 0}$ we fit the data to

$$
\frac{k}{k^{*}}= \begin{cases}0 & \left(v_{\mathrm{p}} \leq v_{\mathrm{p} 0}\right), \\ 1-e^{1-v_{\mathrm{p}} / v_{\mathrm{p} 0}} & \left(v_{\mathrm{p}}>v_{\mathrm{p} 0}\right) .\end{cases}
$$

Equation (4) is not derived but a phenomenological relation that captures the key aspects of the data discussed above: (1) $k=0$ at small $v_{\mathrm{p}}$; (2) $k$ increases when $v_{\mathrm{p}}>v_{\mathrm{p} 0}$; (3) $k$ approaches $k^{*}$ as $v_{\mathrm{p}} \rightarrow \infty$. Plotting the data in terms of normalized variables $k / k^{*}$ and $v_{\mathrm{p}} / v_{\mathrm{p} 0}$ scales out the dependencies on $\phi_{0}$ and $\eta_{0}$. The resulting data collapse for the longitudinal front speed ratio $k_{1} / k_{1}^{*}$ is plotted in Fig. 4c. A similar result is obtained for the transverse speed ratio $k_{\mathrm{t}} / k_{\mathrm{t}}^{*}$.

To quantify the anisotropy in front propagation in longitudinal and transverse directions, we plot $k_{1}^{*}$ versus $k_{\mathrm{t}}^{*}$ in Fig. $4 \mathrm{~d}$, using the data from experiments varying $\phi$ (larger packing fraction increases both $k_{1}^{*}$ and $k_{\mathrm{t}}^{*}$ ) and $\eta_{0}$. To a good approximation, all the data follow $k_{1}^{*}=2 k_{\mathrm{t}}^{*}$. Comparison of our data with the data obtained for quasi-2D suspensions ${ }^{12}$ shows excellent agreement as well, except that for higher $k_{\mathrm{t}}$ the ratio $k_{1} / k_{\mathrm{t}}$ slightly exceeds 2 .

\section{Discussion}

Our data in Fig. 3c demonstrate that impact-activated jamming of dense suspensions proceeds without significant increase of $\phi$, and certainly without increasing $\phi$ to values close to $\phi_{\mathrm{J}}$. This rules out earlier explanations based on entering the jammed state via densification of the particle sub-phase ${ }^{5,14}$. Instead, analysis of the flow field shows that the jamming fronts initiated by the impact 

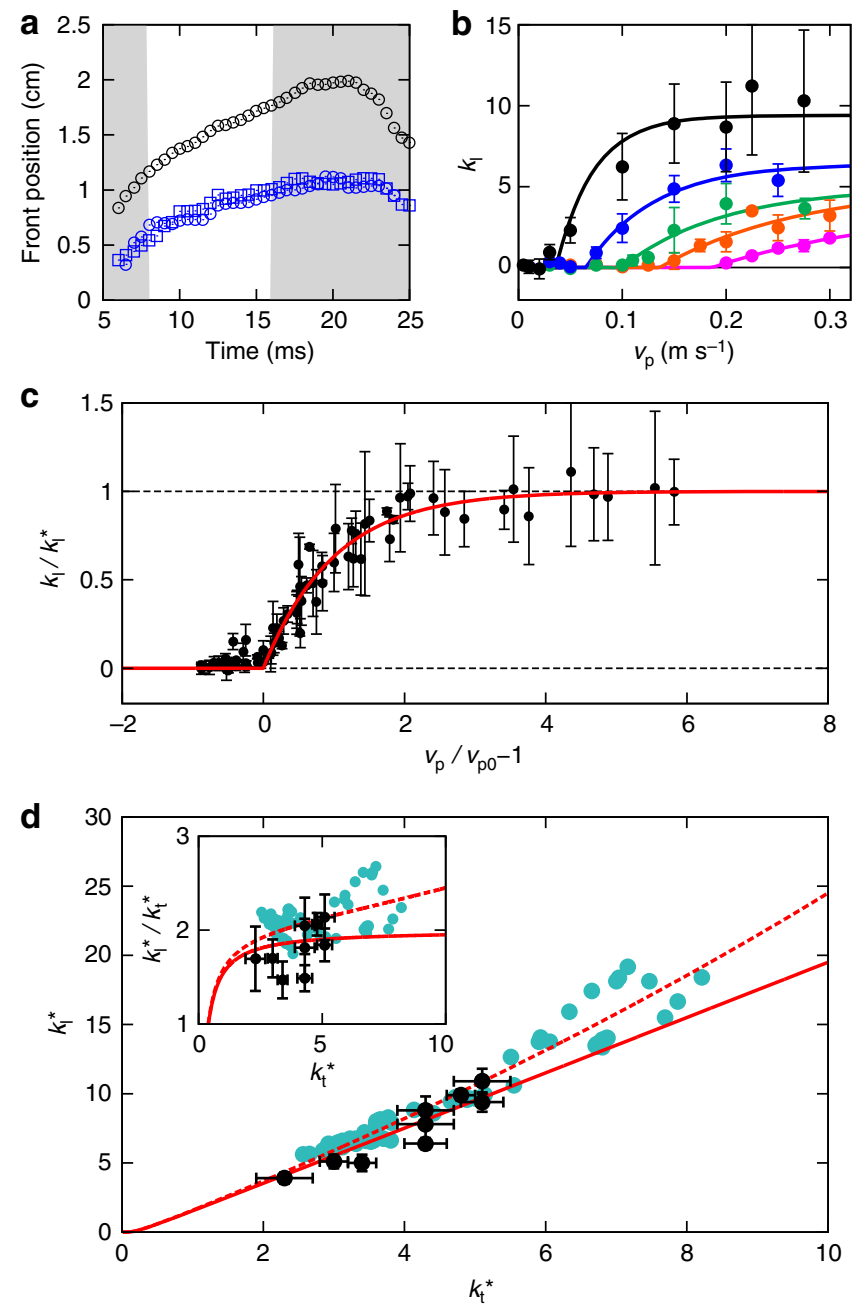

Figure 4 | Propagation of jamming fronts. (a) Front position in longitudinal (black) and transverse (blue circles: right, blue squares: left) directions as functions of time. The impactor touches the suspension surface at time $t=0 \mathrm{~ms}$. The grey shaded background indicates the initial front build-up (left), and the regime where the fronts starts to interact with boundaries and slows down (right). For these data $v_{\mathrm{p}}=200 \mathrm{~mm} \mathrm{~s}^{-1}$, $\phi=0.460$. (b) Normalized front propagation speed $k_{\mathrm{l}}$ in longitudinal direction as function of $v_{\mathrm{p}}$ for different $\phi$ (magenta: 0.439 , orange: 0.453 , green: 0.460 , blue: 0.474 , black: 0.498 ). All data are for suspending liquid viscosity $\eta_{0}=4.6 \pm 0.2 \mathrm{mPa} \cdot \mathrm{s}$. Error bars show the standard deviation of three measurements. The same data plotted in log-linear scale are shown in Supplementary Fig. 3. (c) Front speed $k_{l} / k_{1}^{*}$ normalized by its asymptotic value as function of impactor speed $v_{\mathrm{p}}$ normalized by threshold speed $v_{\mathrm{pO}}$. Data from experiments with different $\phi$ and $\eta_{0}$ collapse onto a master curve fit by equation (4) (solid red line). (d) Relationship between the asymptotic front speeds $k_{1}^{*}$ and $k_{\mathrm{t}}^{*}$ in longitudinal and transverse direction, respectively. Data from both quasi-2D (ref. 12) (turquoise) and 3D (black) impact experiments are shown. The solid red line is the prediction from equation (5). The slope approaches 2 as $k_{\mathrm{t}}^{*}$ increases. The dashed red line is a modified version of the model, which includes a small strain anisotropy $\delta$, here plotted using a value of $\delta=0.01$. Error bars are the asymptotic s.e. from the fittings of each $k-v_{p}$ curve with equation (4).

coincide with the location of the maxima in local shear rate (Fig. 2d,e). Altogether, these two findings provide strong evidence for dynamic shear jamming: the impact triggers propagating fronts that locally create sufficient shear to reorganize particles into (anisotropic) jammed configurations without changing the average packing fraction. There are several implications of the shear jamming scenario for suspensions and several differences from dry granular systems, both of which we discuss next.

To start, we examine the stress. In a dry granular system, stress is sustained only via direct contact between particles. By contrast, in a dense suspension stress can also be transmitted without contact via lubrication forces. Thus, while in dry granular systems there is only a single characteristic stress scale for entry into the shear-jammed regime ${ }^{16}$, for a suspension the situation can be more complex. A number of theoretical models ${ }^{3}$, simulations ${ }^{2,26}$ and experiments ${ }^{27-29}$ have recently suggested that lubrication breaks down and particles start to experience frictional interactions beyond a local stress threshold $\sigma_{1}$. Thus, for stress levels below $\sigma_{1}$ the suspension behaves fluid-like, while above $\sigma_{1}$ the system can be thought to behave more like a frictional granular system, that is, enter a fragile regime before crossing over into the shear-jammed regime at a second characteristic stress level $\sigma_{2}$ (ref. 16). Within this picture, we associate the transition at $v_{\mathrm{p} 0}$ with the situation where the stress levels at the leading edge of the jamming front have reached $\sigma_{1}$ and are large enough for frictional interactions to occur. Thus, when $v_{\mathrm{p}}<v_{\mathrm{p} 0}$ the suspension is in the lubrication regime, but when $v_{\mathrm{p}}>v_{\mathrm{p} 0}$ it transitions into a fragile state with behaviour intermediate between solid and fluid ${ }^{16,30,31}$, as frictional contacts start percolating through the system to form a load-bearing network, eventually reaching a shear-jammed state as $v_{\mathrm{p}}$ increases further. We point out that a non-zero shear modulus is not strictly necessary for the front to propagate (with $k>0$ ). The front will propagate as long as it transforms the initially liquid-like suspension into a state with sufficiently large viscosity. However, we know that this state behaves solid-like, while interacting with a system boundary ${ }^{5,6,10,12}$. Thus, how the shear modulus evolves in the region behind the jamming front before a system-spanning jammed state has been established remains an open question.

The stress-based argument also provides an explanation of the relaxation or 'melting' of the jammed region when the impact stops. During front propagation the stress inside the jammed region is sustained by the inertia of the suspension in the shear zone ahead of the jammed region. When the motion of the impactor stops, the shear zone disappears and the stress applied on the boundary of the jammed suspension falls below $\sigma_{1}$, insufficient to sustain frictional interactions between particles and therefore any network of force chains that could generate a yield stress and support a load. As a result, the suspension returns to the lubrication regime.

However, while necessary, the existence of threshold stress levels is not sufficient to explain the asymptotic front speed $k^{*}$ at high $v_{\mathrm{p}}$ and the seemingly universal anisotropy in front propagation, expressed by the ratio $k_{1}^{*} / k_{\mathrm{t}}^{*} \approx 2$. Particles also need to move out of an initial uniform isotropic distribution and reorganize under shear into anisotropic structures (force chains) that can support the stress. Such reorganization requires a minimum shear strain $\varepsilon_{\mathrm{c}}$ to engage neighbouring particle layers. As a result, shear jamming happens only when stress and strain both reach their threshold values. In a quasi-static granular system $^{16,17}$ the threshold strain only matters when the shearjammed state is prepared or when the shear is reversed. In the dynamic system considered here, the front continues to propagate into unperturbed suspension, and therefore, the front advances by applying strain $\varepsilon_{c}$ locally during the whole process of front propagation.

For dense suspensions in the high $v_{\mathrm{p}}$ regime, where the stress threshold is clearly exceeded, we can show that $k^{*}$ is governed by $\varepsilon_{\mathrm{c}}$ and that, in fact, the front speed anisotropy is a direct consequence of the existence of a strain threshold. As described 
above, the suspension experiences pure shear in the longitudinal direction and simple shear in the transverse direction. In 2D, we can directly compare the two types of shear using the positive eigenvalues of the shear rate tensors, treating the propagation of the front in the longitudinal and transverse directions as two effectively $1 \mathrm{D}$ problems. We now assume that a suspension element jams when the shear strain it experiences reaches $\varepsilon_{c}$, irrespective of propagation direction. This leads to the following relations between $k_{1}^{*}, k_{\mathrm{t}}^{*}$ and $\varepsilon_{\mathrm{c}}$ (see Methods section):

$$
k_{\mathrm{t}}^{*}=1 /\left(2 \varepsilon_{\mathrm{c}}\right), k_{\mathrm{l}}^{*}=1 /\left(e^{\varepsilon_{\mathrm{c}}}-1\right),
$$

and

$$
k_{1}^{*}=\frac{1}{e^{1 /\left(2 k_{\mathrm{t}}^{*}\right)}-1} .
$$

Equation (6) is plotted in Fig. 4d. For small $\varepsilon_{\mathrm{c}}$ we find from equation (5) that $k_{1}^{*} \approx 2 k_{\mathrm{t}}^{*}=1 / \varepsilon_{c}$. In other words, the anisotropy ratio of 2 in the normalized front speeds originates from the factor $1 / 2$ in the non-diagonal terms of the shear rate tensor, which in turn arises because simple shear can be decomposed into a combination of pure shear and solid body rotation. In $3 \mathrm{D}$, it is not possible to quantify the effects of pure shear and simple shear via the same approach (see Methods section). However, one possible solution is to use the 'strain intensity' $\mathcal{D}$ suggested by Ramsay and Huber ${ }^{32}$, which provides a scalar measure of the relative strength of the two types of shear. As we show in the Methods section, this leads to a ratio $k_{1}^{*} / k_{\mathrm{t}}^{*} \approx 3 / \sqrt{2} \approx 2.12$, very close to the value for the $2 \mathrm{D}$ case. Therefore, an anisotropy ratio $\approx 2$ agrees very well with the experimental data for both quasi-2D (ref. 12) and 3D systems within our measurement precision.

With increasing packing fraction $\phi$ the average distance between particles decreases and we expect the strain threshold $\varepsilon_{\mathrm{c}}$ to decrease as well, which agrees qualitatively with the measurements of $\varepsilon_{\mathrm{c}}$ in dry granular systems ${ }^{16}$. Via equation (5) this explains the increase in $k^{*}$ with $\phi$ seen in Fig. $4 \mathrm{~b}$ : since it takes less strain to reorganize the particles into a shear-jammed network the front will propagate faster for given impactor velocity. We point out that equation (1), which formalizes such relationship between packing fraction and front speed, appears to capture the overall trend qualitatively. However, this seems fortuitous, since equation (1) was based on the assumption that the moving front significantly increases the packing fraction, in fact driving it up all the way to $\phi_{\mathrm{J}}$, which we now can rule out in our system. In addition, equation (1) cannot predict the observed propagation anisotropy. One of the outstanding tasks therefore is to develop a model for $k^{*}(\phi)$ that is based on jamming by shear rather than densification.

An interesting aspect of the data in Fig. $4 \mathrm{~d}$ is the deviation from the anisotropy ratio $\approx 2$ at large $k^{*}$ values or, equivalently, large packing densities. This is most apparent in the data available for the quasi-2D system and it indicates that the longitudinal speed becomes faster. We speculate that this may be connected to a breakdown of the assumption of an isotropic threshold $\varepsilon_{c}$. For example, if the impact were to introduce a small amount of compression of the particle sub-phase in longitudinal direction, $\varepsilon_{\mathrm{c}}$ would be reduced in that direction. This effect would become increasingly significant at large $\phi$. We can model this by introducing a correction $\delta$ so that

$$
k_{1}^{*}=1 /\left(e^{\varepsilon_{\mathrm{c}}-\delta}-1\right) .
$$

Using $\varepsilon_{\mathrm{c}}=1 /\left(2 k_{\mathrm{t}}^{*}\right)$ and $\delta \approx 0.01$ we can reproduce the trend in Fig. $4 \mathrm{~d}$ well (dotted red line). However, we point out that this is just the simplest way to account for the trend in the data and there might be other reasons for the deviation.
Taken together, these results provide important insights into the mechanism responsible for impact-induced solidification of dense suspensions. The finding that the packing fraction does not increase measurably during impact, together with the observation of strong shear at the leading edge of the propagating solidification fronts, rules out jamming via densification as the dominant mechanism and points to jamming by shear (densification is likely to play a significant role at much larger impact velocities, when the interstitial liquid's compressibility can no longer be neglected ${ }^{15}$ ). In dense suspensions this introduces a new stress scale or, equivalently, an impact velocity threshold, which we associate with the breakdown of lubrication films between particles and the onset of frictional interactions $s^{2,3,27-29}$. Behind the front, these frictional interactions create a dynamically shear-jammed region (corresponding to fragile and/or shearjammed states in refs 3,16). Further support for the shearjamming scenario comes from the observation of anisotropic front propagation, where we can relate the fact that the fronts propagate longitudinally twice as fast as in transverse direction to an equivalent factor in the ability to transmit shear strain. We point out that for dynamic shear jamming, both shear stress and strain need to exceed threshold values, and the critical shear strain determines the front propagation speed.

\section{Methods}

Experimental set-up. The ultrasound measurements and imaging were performed with a Verasonics Vantage 128 system. The sample container was 3D-printed from ultraviolet-cured resin ('Vero White Plus', Objet Geometries Inc.) whose acoustic impedance matched the suspensions we studied. The inner diameter of the container was $100 \mathrm{~mm}$ and the typical depth $H$ of the suspension was 25 to $35 \mathrm{~mm}$ This insured that the front reached the bottom before it interacted with the side wall. The impactor was a cylinder of diameter of 6 or $10 \mathrm{~mm}$, driven by a computer controlled linear actuator (SCN5, Dyadic Systems) and equipped with a force sensor (DLC101, Omega). The ultrasound transducer (Philips L7-4; 128 independent elements; total width of $38 \mathrm{~mm}$ ) contacted the bottom of the container through a thin layer of ultrasound gel. The ultrasound system was triggered as the impactor approached the surface; images were taken at a frame rate of 10 to 10,000 frames per second, adjusted according to $v_{\mathrm{p}}$. The spatial resolution of the ultrasound was limited by the wavelength, about $0.4 \mathrm{~mm}$ in our experiments.

Suspensions. The cornstarch (Ingredion) was stored in the lab environment at $22.5 \pm 0.5^{\circ} \mathrm{C}$ and $51 \pm 2 \%$ relative humidity. Individual particles had a diameter of 5-30 $\mu \mathrm{m}$ (refs 5,33). The suspending solvent was a solution of $\mathrm{CsCl}$, glycerol and water. Its viscosity $\eta_{0}$ was adjusted by the mass ratio of glycerol and water, and its density was matched to that of the cornstarch particles by adjusting the mass ratio of CsCl. The density of the particles was $\rho_{\mathrm{cs}}=(1.63 \pm 0.01) \times 10^{3} \mathrm{~kg} \mathrm{~m}^{-3}$ as measured by density matching. To calculate the packing fraction $\phi$, an accurate determination of the volume occupied by particles and interstitial liquid is required. For cornstarch this is difficult because the particles are porous and they already contain some moisture before they are dispersed in the suspending solvent. Therefore, often a value $\phi_{\mathrm{m}}$ based on the mass fractions before mixing is quoted $^{4,5,7}$, which is proportional to $\phi$. For example, in Fig. $4 \mathrm{~b}$, the $\phi_{\mathrm{m}}$ values were $0.390,0.402,0.409,0.425$ and 0.444 . To obtain the actual packing fraction, we account for a small fraction $\alpha$ of suspending liquid that is wicked up by the porous particles and write $\phi=(1+\alpha) \phi_{\mathrm{v}}$, where $\phi_{\mathrm{v}}$ is the material volume packing fraction ${ }^{34,35}$. When calculating $\phi_{\mathrm{v}}$, we considered the moisture in the cornstarch particles. We assume the moisture is pure water and its mass ratio in cornstarch is $\beta$ in our lab environment. This leads to

$$
\phi_{\mathrm{v}}=\frac{(1-\beta) m_{\mathrm{cs}} / \rho_{\mathrm{cs}}}{(1-\beta) m_{\mathrm{cs}} / \rho_{\mathrm{cs}}+m_{\mathrm{l}} / \rho_{\mathrm{l}}+\beta m_{\mathrm{cs}} / \rho_{\mathrm{w}}},
$$

where $m_{\mathrm{cs}}$ is the mass of cornstarch particles, $m_{1}$ is the mass of the solvent liquid, $\rho_{\mathrm{l}}$ is the density of the solvent and $\rho_{\mathrm{w}}$ is the density of pure water. In this paper we use $\alpha=0.3$ and $\beta=0.11$ by estimation. Changing of these numbers will not affect the conclusions we make.

Since air bubbles mixed in the suspension scatter ultrasound signals significantly, the suspensions were debubbled before using. To keep $\phi$ fixed during debubbling, we filled the suspensions into sealed syringes and then lowered the pressure by pulling the plungers. The syringe walls were tapped gently to help bubbles separate from the suspension. After debubbling a small amount of suspension was used to measure the speed of sound $c$ as required for image reconstruction. For imaging the flow field, a small amount of air bubbles were added back to the debubbled suspensions to act as tracer particles. This was done by slowly stirring the suspension, then tilting and slowly rotating the container till 
the bubbles were uniformly distributed. We verified that the amount of bubbles did not suppress the penetration of the ultrasound in the suspensions and produced a negligible change in the speed of sound. We also determined that the effect of the bubbles on the suspension viscosity is limited (Supplementary Fig. 4). Between successive impact experiments the suspension was relaxed by gently shaking and rotating the container.

Data acquisition and analysis. Once triggered, the ultrasound system made several hundred acquisitions consecutively. In one acquisition each of the 128 transducer elements transmitted the same ultrasonic pulse at the same time and received an individual reflected time series. The pulse was a $5-\mathrm{MHz}$ sinusoidal wave modulated by a Gaussian profile (Gaussian envelope) for a pulse length of 6 periods. From the time series received by the transducer and using the previously measured speed of sound $c$ we reconstructed B-mode images (using brightness to represent the echo signal amplitude) ${ }^{25}$ that captured the positions of the tracer particles (air bubbles) in the suspension. Given our finding that $c$ does not change measurably during the impact, the image reconstruction process does not need to account for spatial or temporal variations in $c$. By tracking the tracer bubble displacements with a particle imaging velocimetry (PIV) algorithm, we obtained a 2D flow field from within the bulk of the suspension.

Change of packing fraction measurements. The experimental set-up was identical to the one illustrated in Fig. 1 and a schematic illustration is shown in Fig. 3b. The impactor started from the surface of the suspension and pushed down a distance $z_{\mathrm{p}}$. The position of the impactor was measured with a high-speed camera (Phantom V9, Vision Research). The ultrasound measured the time of flight $T$ of the signal transmitted from the bottom, reflected by the impactor and sent back to the bottom. Thus the average speed of sound $\bar{c}$ along this path is

$$
\bar{c}=2 \frac{H-z_{\mathrm{p}}}{T} .
$$

We started with experiments at a low $v_{\mathrm{p}}\left(5 \mathrm{~mm} \cdot \mathrm{s}^{-1}\right)$ to measure the speed of sound in the liquid-like, unjammed suspension, where $\bar{c}=c\left(\phi_{0}\right)$. Define $T_{0}$ as the initial time of flight when $z_{\mathrm{p}}=0 \mathrm{~mm}$, we have $H=c\left(\phi_{0}\right) T_{0} / 2$, and from this

$$
c\left(\phi_{0}\right)=\frac{2 z_{\mathrm{p}}}{T_{0}-T} .
$$

The initial packing fraction $\phi_{0}$ in these experiments was 0.48 . The liquid was a mixture of $44.3 \% \mathrm{CsCl}, 27.8 \%$ glycerol and $27.8 \%$ water by mass, with $\eta_{0}=4.6 \mathrm{mPa} \cdot \mathrm{s}$ and $\rho=1.63 \times 10^{3} \mathrm{~kg} \cdot \mathrm{m}^{-3}$. From six measurements we obtain $c\left(\phi_{0}\right)=1939.2 \pm 4.6 \mathrm{~m} \mathrm{~s}^{-1}$ and $H=c\left(\phi_{0}\right) T_{0} / 2=34.1 \pm 0.1 \mathrm{~mm}$.

For the high $v_{\mathrm{p}}\left(200 \mathrm{~mm} \mathrm{~s}^{-1}\right)$ experiments we used the value of $H$ measured above and equation (9) to calculate $\bar{c}$. The time of flight now becomes

$$
T=2\left[\frac{H-z_{\mathrm{f}}}{c\left(\phi_{0}\right)}+\frac{z_{\mathrm{f}}-z_{\mathrm{p}}}{c\left(\phi_{0}+\Delta \phi\right)}\right] .
$$

For $\Delta \phi=0, T_{\Delta \phi=0}=2\left(H-z_{\mathrm{p}}\right) / c\left(\phi_{0}\right)$. If $\Delta \phi>0$, there will be a difference between $T$ and $T_{\Delta \phi=0}$, and the difference becomes increasingly large as $z_{\mathrm{f}}$ increases, which leads to an increase in $\bar{c}$ according to equation (9).

Derivation of equation 5 in 2D. For an idealized 2D system we define a Cartesian coordinate with $x$ axis in the transverse and $y$ axis in the longitudinal direction. To obtain the relation between the strain threshold $\varepsilon_{\mathrm{c}}$ and the normalized front speeds $k$ we consider how much shear strain a suspension element experiences when it accelerates from $u_{y}=0$ to $u_{y}=v_{\mathrm{p}}$. We consider the propagation in the transverse and longitudinal directions separately as two quasi-1D problems. Exemplary sketches of the velocity profiles are provided in Supplementary Fig. 5 . The experimental data did not show a significant change in front width, so here we assume the shape of the front does not change during propagation. In this case the velocity profiles can be expressed as

$$
u_{y}(x, t)=f_{\mathrm{t}}\left(x-v_{\mathrm{ft}} t\right),
$$

in the transverse direction and

$$
u_{y}(y, t)=f_{1}\left(y-v_{\mathrm{ff}} t\right),
$$

in the longitudinal direction. In both the equations $t$ is time, $v_{\mathrm{ft}}$ and $v_{\mathrm{fl}}$ are front propagation speeds. $f_{\mathrm{t}}(X)$ and $f_{1}(X)$ are functions that satisfy $f_{\mathrm{t}}=f_{1}=v_{\mathrm{p}}$ as $X \rightarrow-\infty$ and $f_{\mathrm{t}}=f_{1}=0$ as $X \rightarrow+\infty$.

On either side of the impactor the front propagates in transverse direction and the front speed $v_{\mathrm{ft}}=k_{\mathrm{t}} v_{\mathrm{p}}$, while the suspension itself is sheared longitudinally by the advancing front. The acceleration of a suspension element is then

$$
\frac{\mathrm{D} u_{y}(x, t)}{\mathrm{D} t}=\frac{\partial f_{\mathrm{t}}}{\partial t}=-k_{\mathrm{t}} v_{\mathrm{p}} f_{\mathrm{t}}^{\prime}=-k_{\mathrm{t}} v_{\mathrm{p}} \frac{\partial u_{y}}{\partial x},
$$

where $D / D t$ is the material derivative and $f_{t}^{\prime}=d f_{t}(X) / d X$. Below the impactor there are two differences: one is that the suspension element now moves along the propagation direction of the front and the other is that $v_{\mathrm{fl}}=\left(k_{1}+1\right) v_{\mathrm{p}}$ as defined in equation (3). The acceleration then becomes

$$
\begin{aligned}
\frac{D u_{y}(y, t)}{D t} & =\frac{\partial f_{1}}{\partial t}+\left(u_{y} \frac{\partial}{\partial y}\right) f_{1}=\left[u_{y}-\left(k_{1}+1\right) v_{\mathrm{p}}\right] f_{1}^{\prime} \\
& =\left[u_{y}-\left(k_{1}+1\right) v_{\mathrm{p}}\right] \frac{\partial u_{y}}{\partial y} .
\end{aligned}
$$

Now we look at the relation between the local shear rate $\dot{\varepsilon}$ and the velocity gradient. In general, for an incompressible $2 \mathrm{D}$ fluid the shear rate tensor is

$$
\dot{\varepsilon}=\left[\begin{array}{cc}
\frac{\partial u_{x}}{\partial x} & \frac{1}{2}\left(\frac{\partial u_{x}}{\partial y}+\frac{\partial u_{y}}{\partial x}\right) \\
\frac{1}{2}\left(\frac{\partial u_{x}}{\partial y}+\frac{\partial u_{y}}{\partial x}\right) & \frac{\partial u_{y}}{\partial y}
\end{array}\right],
$$

where $\frac{\partial u_{x}}{\partial x}=-\frac{\partial u_{y}}{\partial y}$. From experimental observation we have $\frac{\partial u_{x}}{\partial y} \ll \frac{\partial u_{y}}{\partial x}$. For the transverse direction, where simple shear dominates, the diagonal terms vanish and the shear rate tensor becomes

$$
\dot{\varepsilon}_{\mathrm{t}}=\left[\begin{array}{cc}
0 & \frac{1}{2} \frac{\partial u_{y}}{\partial x} \\
\frac{1}{2} \frac{\partial u_{y}}{\partial x} & 0
\end{array}\right]
$$

while for pure shear in the longitudinal direction the off-diagonal terms vanish and we have

$$
\dot{\varepsilon}_{1}=\left[\begin{array}{cc}
-\frac{\partial u_{y}}{\partial y} & 0 \\
0 & \frac{\partial u_{y}}{\partial y}
\end{array}\right] .
$$

In either case the matrix has two eigenvalues with the same magnitude but opposite sign, and the eigenvalues represent the shear rate on the principal axes. Thus, we can represent the shear intensities by the tensors' positive eigenvalues: $\dot{\varepsilon}_{1}=\left|\frac{\partial u_{y}}{\partial y}\right|=-\frac{\partial u_{y}}{\partial y}$ and $\dot{\varepsilon}_{\mathrm{t}}=\frac{1}{2}\left|\frac{\partial u_{y}}{\partial x}\right|=-\frac{1}{2} \frac{\partial u_{y}}{\partial x}$.

Using the velocity gradient, we relate the local shear rate with the acceleration of the element:

$$
\begin{gathered}
\dot{\varepsilon}_{\mathrm{t}}=\frac{1}{2} \frac{1}{k_{\mathrm{t}} v_{\mathrm{p}}} \frac{\mathrm{D} u_{y}}{\mathrm{D} t}, \\
\dot{\varepsilon}_{\mathrm{l}}=\frac{1}{\left(k_{\mathrm{l}}+1\right) v_{\mathrm{p}}-u_{y}} \frac{D u_{y}}{D t} .
\end{gathered}
$$

Consequently, the total shear strain $\varepsilon$ a suspension element experiences before jamming is:

$$
\varepsilon_{\mathrm{t}}=\int_{0}^{\infty} \dot{\varepsilon}_{\mathrm{t}} d t=\int_{0}^{v_{\mathrm{p}}} \frac{1}{2 k_{\mathrm{t}} v_{\mathrm{p}}} d u_{y}=\frac{1}{2 k_{\mathrm{t}}},
$$

and

$$
\varepsilon_{1}=\int_{0}^{\infty} \dot{\varepsilon}_{1} d t=\int_{0}^{v_{\mathrm{p}}} \frac{1}{\left(k_{1}+1\right) v_{\mathrm{p}}-u_{y}} d u_{y}=\ln \left(\frac{k_{1}+1}{k_{1}}\right) .
$$

Equation (19) gives $\varepsilon_{1} \approx 1 / k_{1}$ for $k_{1} \gg 1$. If we assume the strain threshold to jamming $\varepsilon_{\mathrm{c}}$ is isotropic, then $k_{\mathrm{t}}=1 /\left(2 \varepsilon_{\mathrm{c}}\right)$ and $k_{\mathrm{l}}=1 /\left(e^{\varepsilon_{\mathrm{c}}}-1\right)$.

Relation between $\boldsymbol{k}_{\mathbf{1}}$ and $\boldsymbol{k}_{\mathbf{t}}$ in 3D. In $3 \mathrm{D}$, the shear rate tensor is shown in equation (2). In the longitudinal direction, pure shear dominates and the shear rate tensor is

$$
\dot{\varepsilon}_{1}=\left[\begin{array}{ccc}
\frac{\partial u_{r}}{\partial r} & 0 & 0 \\
0 & \frac{u_{r}}{r} & 0 \\
0 & 0 & \frac{\partial u_{z}}{\partial z}
\end{array}\right],
$$

where $\frac{\partial u_{r}}{\partial r} \approx \frac{u_{r}}{r}$ and $\frac{\partial u_{r}}{\partial r}+\frac{u_{r}}{r}+\frac{\partial u_{z}}{\partial z}=0$. In the transverse direction simple shear dominates. This gives

$$
\dot{\varepsilon}_{\mathrm{t}} \approx\left[\begin{array}{ccc}
0 & 0 & \frac{1}{2} \frac{\partial u_{z}}{\partial r} \\
0 & 0 & 0 \\
\frac{1}{2} \frac{\partial u_{z}}{\partial r} & 0 & 0
\end{array}\right],
$$

where we have used $\frac{\partial u_{r}}{\partial z} \ll \frac{\partial u_{z}}{\partial r}$. Note that though the system is $3 \mathrm{D}$ simple shear only operates in the $r z$ plane while leaving the azimuthal direction invariant. The eigenvalues become $\dot{e}_{1}=\left\{-\frac{1}{2} \frac{\partial u_{z}}{\partial z},-\frac{1}{2} \frac{\partial u_{z}}{\partial z}, \frac{\partial u_{z}}{\partial z}\right\}$ and $\dot{e}_{\mathrm{t}}=\left\{-\frac{1}{2} \frac{\partial u_{z}}{\partial r}, 0, \frac{1}{2} \frac{\partial u_{z}}{\partial r}\right\}$. Unlike the $2 \mathrm{D}$ case, we cannot simply use a positive eigenvalue to represent the shear intensity. However, we can define infinitesimal strains $e_{i}(i=1,2,3)$ along the three principal axes and rank-order them according to $e_{1}>e_{2}>e_{3}$. Following the definition given in ref. 32 , the 'strain intensity' $\mathcal{D}$ is

$$
\begin{aligned}
\mathcal{D} & =\sqrt{\left(\ln \frac{1+e_{1}}{1+e_{2}}\right)^{2}+\left(\ln \frac{1+e_{2}}{1+e_{3}}\right)^{2}} \\
& \approx \sqrt{\left(e_{1}-e_{2}\right)^{2}+\left(e_{2}-e_{3}\right)^{2}} .
\end{aligned}
$$

For pure shear in the longitudinal direction $e_{1}=e_{2}=-e_{3} / 2$ and $\dot{e}_{3}=\frac{\partial u_{2}}{\partial z}$, so $\mathcal{D}_{1} \approx \frac{3}{2}\left|e_{3}\right|$, which leads to $\dot{\mathcal{D}}_{1} \approx-\frac{3}{2} \frac{\partial u_{z}}{\partial z}$. For simple shear in the transverse direction $e_{1}=-e_{3}, e_{2}=0$ and $\dot{e}_{3}=\frac{1}{2} \frac{\partial u_{2}}{\partial r}$. This leads to $\mathcal{D}_{\mathrm{t}} \approx \sqrt{2}\left|e_{3}\right|$, and therefore 
$\dot{\mathcal{D}}_{\mathrm{t}} \approx-\frac{\sqrt{2}}{2} \frac{\partial u_{z}}{\partial r}$. Following the procedure for the $2 \mathrm{D}$ case we have

$$
\begin{gathered}
\dot{\mathcal{D}}_{\mathrm{t}}=\frac{\sqrt{2}}{2} \frac{1}{k_{\mathrm{t}} v_{\mathrm{p}}} \frac{D u_{z}}{D t}, \\
\dot{\mathcal{D}}_{\mathrm{l}}=\frac{3}{2} \frac{1}{\left(k_{1}+1\right) v_{\mathrm{p}}-u_{z}} \frac{D u_{z}}{D t} .
\end{gathered}
$$

Integration then leads to

$$
\mathcal{D}_{\mathrm{t}}=\frac{\sqrt{2}}{2} \frac{1}{k_{\mathrm{t}}}, \mathcal{D}_{\mathrm{l}}=\frac{3}{2} \ln \left(\frac{k_{\mathrm{l}}+1}{k_{\mathrm{l}}}\right) .
$$

Now we again assume that the system shear-jams when $\mathcal{D}$ reaches a threshold strain value $\mathcal{D}_{\mathfrak{c}}$, independent of the type of shear it experiences. From this we find

$$
k_{1}^{*}=\frac{1}{e^{\sqrt{2} /\left(3 k_{\mathrm{t}}^{*}\right)}-1} .
$$

and $k_{1}^{*} / k_{\mathrm{t}}^{*} \approx 3 / \sqrt{2} \approx 2.12$ for large $k$.

Data availability. The data that support the findings of this study are available from the corresponding author on request.

\section{References}

1. Barnes, H. A. Shear-thickening ('dilatancy') in suspensions of nonaggregating solid particles dispersed in Newtonian liquids. J. Rheol. 33, 329-366 (1999).

2. Seto, R., Mari, R., Morris, J. F. \& Denn, M. M. Discontinuous shear thickening of frictional hard-sphere suspensions. Phys. Rev. Lett. 111, 218301 (2013).

3. Wyart, M. \& Cates, M. E. Discontinuous shear thickening without inertia in dense non-Brownian suspensions. Phys. Rev. Lett. 112, 098302 (2014).

4. Brown, E. \& Jaeger, H. M. Shear thickening in concentrated suspensions: phenomenology, mechanisms and relations to jamming. Rep. Prog. Phys. 77, 046602 (2014)

5. Waitukaitis, S. R. \& Jaeger, H. M. Impact-activated solidification of dense suspensions via dynamic jamming fronts. Nature 487, 205-209 (2012).

6. Roché, M., Myftiu, E., Johnston, M. C., Kim, P. \& Stone, H. A. Dynamic fracture of nonglassy suspensions. Phys. Rev. Lett. 110, 148304 (2013).

7. Mukhopadhyay, S., Allen, B. \& Brown, E. A shear thickening transition in concentrated suspensions under impact. Preprint at http://arxiv.org/abs/ 1407.0719 (2014).

8. Liu, B., Shelley, M. \& Zhang, J. Focused force transmission through an aqueous suspension of granules. Phys. Rev. Lett. 105, 188301 (2010).

9. von Kann, S., Snoeijer, J. H., Lohse, D. \& van der Meer, D. Nonmonotonic settling of a sphere in a cornstarch suspension. Phys. Rev. E 84, 060401 (2011).

10. Peters, I. R., Majumdar, S. \& Jaeger, H. M. Direct observation of dynamic shear jamming in dense suspensions. Nature 532, 214-217 (2016).

11. Smith, M. I., Besseling, R., Cates, M. E. \& Bertola, V. Dilatancy in the flow and fracture of stretched colloidal suspensions. Nat. Commun.. 1, 114 (2010).

12. Peters, I. R. \& Jaeger, H. M. Quasi-2D dynamic jamming in cornstarch suspensions: visualization and force measurements. Soft Matter 10, 6564-6570 (2014).

13. Liu, A. J. \& Nagel, S. R. Jamming is not just cool any more. Nature 396, 21-22 (1998).

14. Waitukaitis, S. R., Roth, L. K., Vitelli, V. \& Jaeger, H. M. Dynamic jamming fronts. Europhys. Lett. 102, 44001 (2013).

15. Petel, O. E., Ouellet, S., Loiseau, J., Frost, D. L. \& Higgins, A. J. A comparison of the ballistic performance of shear thickening fluids based on particle strength and volume fraction. Int. J. Impact Eng. 85, 83-96 (2015).

16. Bi, D., Zhang, J., Chakraborty, B. \& Behringer, R. P. Jamming by shear. Nature 480, 355-358 (2011).

17. Kumar, N. \& Luding, S. Memory of jamming and multiscale flow in soft and granular matter. Granular Matter. Preprint at http://arxiv.org/abs/1407.6167 (2016).

18. Jia, X., Caroli, C. \& Velicky, B. Ultrasound propagation in externally stressed granular media. Phys. Rev. Lett. 82, 1863 (1999).

19. Khidas, Y. \& Jia, X. Anisotropic nonlinear elasticity in a spherical-bead pack: influence of the fabric anisotropy. Phys. Rev. E 81, 021303 (2010).

20. Ouriev, B. \& Windhab, E. J. Rheological study of concentrated suspensions in pressure-driven shear flow using a novel in-line ultrasound Doppler method. Exp. Fluids 32, 204-211 (2002).
21. Ouriev, B. \& Windhab, E. J. Novel ultrasound based time averaged flow mapping method for die entry visualization in flow of highly concentrated shear-thinning and shear-thickening suspensions. Meas. Sci. Technol. 14, 140-147 (2003).

22. Urick, R. J. A sound velocity method for determining the compressibility of finely divided substances. J. Appl. Phys. 18, 983 (1947).

23. McClements, D. J. \& Povey, M. J. W. Ultrasound velocity as a probe. Adv. Colloid Interface Sci. 27, 285-316 (1987).

24. Johnson, B. L., Holland, M. R., Miller, J. G. \& Katz, J. I. Ultrasonic attenuation and speed of sound of cornstarch suspensions. J. Acoust. Soc. Am. 133, 1399-1403 (2013).

25. Cobbold, R. S. C. Foundations of Biomedical Ultrasound (Oxford Univ. Press, 2007).

26. Rognon, P. G., Einav, I. \& Gay, C. Flowing resistance and dilatancy of dense suspensions: lubrication and repulsion. J. Fluid Mech. 689, 75-96 (2011).

27. Fernandez, N. et al. Microscopic Mechanism for Shear Thickening of NonBrownian Suspensions. Phys. Rev. Lett. 111, 108301 (2013).

28. Lin, N. Y. C. et al. Hydrodynamic and contact contributions to continuous shear thickening in colloidal suspensions. Phys. Rev. Lett. 115, 228304 (2015).

29. Guy, B. M., Hermes, M. \& Poon, W. C. K. Towards a unified description of the rheology of hard-particle suspensions. Phys. Rev. Lett. 115, 088304 (2015).

30. Cates, M. E., Wittmer, J. P., Bouchaud, J. P. \& Claudin, P. Jamming force chains and fragile matter. Phys. Rev. Lett. 81, 1841 (1998).

31. Vitelli, V. \& van Hecke, M. Marginal matters. Nature 480, 325-326 (2011).

32. Ramsay, J. G. \& Huber, M. I. The Techniques of Modern Structural Geology Vol. 1Academic Press Inc.1st edition, 1983).

33. BeMiller, J. \& Whistler, R. Starch: Chemistry and Technology (Academic Press Inc.3rd edn, 2009).

34. Sair, L. \& Fetzer, W. R. Water sorption by starches. Ind. Eng. Chem 36, 205-208 (1944).

35. Hellman, N. H. \& Melvin, E. H. Surface area of starch and its role in water sorption. J. Am. Chem. Soc. 72, 5186-5188 (1950).

\section{Acknowledgements}

We thank Patrick La Riviere for help with the ultrasound system. We thank Mengfei He, Nicole James, Victor Lee, Sayantan Majumdar, Daniel Szyld, Scott Waitukaitis, Tom Witten, Matthieu Wyart and Qin Xu for insightful discussions. This work was supported by the US Army Research Office through grants W911NF-12-1-0182 and W911NF-16-10078. Additional support was provided by the Chicago MRSEC, which is funded by NSF through grant DMR-1420709.

\section{Author contributions}

All authors designed the experiments and wrote the paper. E.H. performed the experiments and analysed the data. I.R.P. contributed the PIV code.

\section{Additional information}

Supplementary Information accompanies this paper at http://www.nature.com/ naturecommunications

Competing financial interests: The authors declare no competing financial interests.

Reprints and permission information is available online at http://npg.nature.com/ reprintsandpermissions/

How to cite this article: Han, E. et al. High-speed ultrasound imaging in dense suspensions reveals impact-activated solidification due to dynamic shear jamming. Nat. Commun. 7:12243 doi: 10.1038/ncomms12243 (2016).

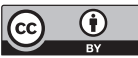

This work is licensed under a Creative Commons Attribution 4.0 International License. The images or other third party material in this article are included in the article's Creative Commons license, unless indicated otherwise in the credit line; if the material is not included under the Creative Commons license, users will need to obtain permission from the license holder to reproduce the material. To view a copy of this license, visit http://creativecommons.org/licenses/by/4.0/

(C) The Author(s) 2016 